\title{
PHYSICAL MEDICINE IN DISEASES OF THE CARDIO-VASCULAR SYSTEM.
}

\author{
By BASIL PARSONS-SMITH, M.D., F.R.C.P. \\ (Physician, National Hospital for Diseases of the Heart, London.)
}

Before reviewing the modern conception of physiotherapy as applied to cardiovascular diseases we should, perhaps, make brief mention of certain of the outstanding considerations which have combined to establish the value of purely physical treatment in circulatory disorders.

Physiological research in recent years has been eminently valuable in that it has analysed the basal physics of the circulation, and it is nowadays generally admitted that, quite apart from medicinal methods, we can remedy a certain number of circulatory discrepancies by the unaided use of carefully chosen physical treatment.

The modern application of such remedies for the relief of cardio-vascular disorders is a natural sequence to the previously well-recognised value of physiotherapy in the maintenance of healthy development and physical fitness, and it is interesting to note that the question of physical training is at the present time a matter of national concern to which the most thorough investigation will be accorded; it is, moreover, worthy of comment that the value of physical therapy was first recognised by certain of the early Grecian and Roman physicians from the writings of whom a moderately continuous reference can be traced through the literature of the middle ages and subsequently down to the present time.

The practice of medical gymnastics and massage was first developed, we read, in the Grecian gymnasia, where the technical treatment was carried out by skilled training masters called pædotribes; historians relate that Herodikus of Selymbria (500 B.C.), being compelled by ill-health to resign active duty as a training master, evolved for himself a course of treatment based on dietary regulations, bathing and exercise, which, although it induced his complete recovery, was criticized by Plato in the following terms:- "By a combination of training and doctoring he found a way of torturing first and chiefly himself and secondly the rest of the world .... by the invention of a lingering death." Hippocrates, the Father of Medicine (460-380 B.c.), was contemporary with Herodikus, and it is abundantly clear that he appreciated the value of exercise and massage, incidentally also he made pioneer observations on the remedial effects of sun treatment. The popularity of physical therapy continued to advance and it is recorded that, in the time of Pompey the Great, Asclipiades (IOO B.C.), an ambitious exponent of the Grecian School, journeyed to Rome and opened an institution in that city for the practice of friction and remedial exercises; he wrote a book dealing with the subject which was mentioned later by Celsus, and he was the first to use hanging beds in which he directed that his patients should be rocked to sleep. Galen (A.D. I30-200) merits a special mention in the history of the development of physical therapy; he described massage and its uses and he appreciated the value of friction in combination with exercise; he wrote several books dealing with the subjects of hygiene and exercise, also the use of baths and massage. Avicenna (A.D. 980I037) was probably one of the most famous Islamic physicians; he had strong convictions in regard to physical therapy and he encouraged athletes in the use of friction after exercise, the object being removal of fatigue products from the muscles; he recommended the application of hot sand, oil baths and various forms of exercises in the direct heat of the sun, all with a view to the promotion of sweating, and the relief of flatulence, lax swellings and dropsies. 
Thomas Sydenham, sometimes referred to as the British Hippocrates, strongly supported the cult of massage and exercises in various diseases; he advocated horse-back riding "to strengthen the blood and spirits," and he even went so far as to lend his own horses to his poorer patients; subsequently Samuel Quellmaltz, being strongly impressed with the curative properties of horse-back riding, invented a suspended rocking horse, which, for various reasons, was preferable to certain of his patients.

The above references will suffice to emphasise certain of the landmarks in the traditional history of physical medicine, not the least noteworthy being the early impetus it was accorded during the Hippocratic era; in its later evolution certain additional methods of treatment, ancillary to friction, massage and exercises, came gradually into prominence; these included hydrotherapy, heliotherapy and electrical treatment, all of which are deserving of special mention in the present context by reason of the status they have gradually acquired in the successful management of cardio-vascular disease.

History relates that bathing in the sea and in the waters of warm springs was encouraged by the Romans, and there is good evidence to show that their bathing establishments were elaborately planned; of the curative value of mineral baths little, however, was known until, after a somewhat crude analysis, they were introduced by Paracelsus, early in the sixteenth century; for the further development of hydrotherapy we are indebted in no small measure to certain of the English physicians who practised at Bath in the I7th century; these included Robert Pierce, an outstanding authority on the subject of balneotherapy, and incidentally a Fellow of the College of Physicians, London, also Sir John Floyero Dr. Edward Baynard and Dr. William Oliver; on their pioneer studies the foundation of hydrotherapy was firmly established and to them and their colleagues should be accorded due credit for the uses of mineral waters in the treatment of diseases the conception and scope of which treatment has gradually matured in British and Continental Spas so that it is nowadays assured the universal approval of the medical profession and favourable recognition by the laity.

It remains to mention certain of the physical forces which, in process of time, have gradually acquired well-merited recognition in the physio-therapeutic field; outstanding examples include heat, light, ultra-violet and electro-magnetic radiations; whilst, however, the very early historians were obviously impressed with the curative value of natural sunshine it was not until the present century that natural heliotherapy (i.e., treatment by heat, light and actinic radiation) was seriously developed and scientifically applied in Switzerland by Oscar Bernhard and Auguste Rollier; to the brilliant researches of these two workers a lasting tribute must be accorded, as also to Niels Ryberg Finsen, the founder of ultraviolet radiation therapy, who not only recognised the curative value of natural sunshine from which the heat rays had been eliminated by means of a quartz filter, but also introduced a method of artificial ultra-violet ray treatment with a $N$ specially designed carbon arc lamp.

Electricity is another of the physical forces that has been extensively used in the treatment of a variety of diseases, and certain forms of electro-therapy have 0 justified their inclusion in the management of cardio-vascular complications.

William Gilbert, a sixteenth century president of the College, was responsible $\stackrel{\mathscr{\Phi}}{\rightarrow}$ for valuable researches, but the first physician who actually used electricity for 0 medical purposes was a German named Kratzenstein, who investigated the subject $\stackrel{\circ}{\circ}$ at Copenhagen in the eighteenth century under the patronage of the King of 
Denmark; others who advanced the application of electro-therapy were Kratzenstein's contemporaries Marat and Galvani, and it is noteworthy that electrical machines were installed in two London hospitals, the Middlesex and St. Bartholomew's, in I767 and I777 respectively; subsequently (I836) an electrical department was instituted at Guy's Hospital under the direction of Dr. Golding Bird, and it is recorded that he read several communications before the College of Physicians, London, on "Electricity and galvanism in relation to physiology and therapeutics," including the indications for electro-magnetic induction currents which a few years earlier (I83I) had been discovered by Michael Faraday; further developments followed, the more important being the work of d'Arsonval and Kellogg on sinusoidal currents, and the investigations of Nikola Tesla, Nernet and Nagelschmidt, which have served as the foundation for the evolution of so-called high frequency or diathermy treatment.

The above-mentioned remedies form the basis of the physiotherapy that is nowadays available for the treatment of cardiac and circulatory disorders, and one can gain some idea of the scope of such treatment in that it has approved indications in the great majority of cardiological problems.

Excluding, for the moment, the purely functional syndromes it is customary to recognise in all heart cases some forms of structural disease and mechanical errors in the circulation secondary thereto; the former are more often than not permanent and irremedial but the latter (i.e., the mechanical discrepancies) can usually be alleviated to some extent and it is in this connection that physical treatment is more especially valuable.

A brief reference to the theories and practice of massage and remedial exercises will suffice to outline certain of the more essential aspects of the subject. Our first consideration should be to appreciate as fundamental the principles relating to the promotion of health by physio-therapeutic methods which have been bequeathed to us through the ages, secondly we should assess the physiological interpretation of the behaviour of the body during muscular exercise, for it is on a knowledge of such basic principles that we must rely for the successful treatment of the unfit, incidentally also for the maintenance of the ideal health and well-being of the general community.

It is common knowledge that during exercise the circulation rate must be accelerated in order that the tissue demands shall be met, and there must obviously be an intimate correlation between the pulmonary ventilation and the circulation rate. A larger cardiac output per minute involves a corresponding increase in the diastolic filling process which the physiologists tell us is determined entirely by the amount of the venous inflow; the latter is increased during exercise by the greater volume of blood returning from the skeletal muscles, incidentally also by the accelerated inflow of blood to the inferior vena cava which develops in consequence of the more vigorous activity of the diaphragm and the raised intra-abdominal pressure with which it is associated.

The natural sequel to a larger venous inflow is an increased output per minute which the heart accomplishes, either by adding to its output per beat or by accelerating its rate of contraction; of these the former, i.e., the increased output per beat, is well explained by Starling's law of the heart which states that the energy set free and the contractile power of the heart muscle are in direct proportion to the initial length of its fibres; in other words the diastolic volume of the heart determines the force of its contraction and the volume of its output per beat; such are the fundamental principles which govern the natural ability of the heart 
to increase its functional efficiency in response to a larger venous return, and it $\frac{2}{0}$ should be emphasised in this connection that exercise normally induces some $c$. degree of physiological dilatation for on this depends the increase of contractile $\vec{\Rightarrow}$ power, which, according to Starling's law, enables the heart to increase its output $\stackrel{5}{\rightarrow}$ in response to a larger venous return. We must realise, however, that whilst stillo obeying Starling's law, the contractile power of the heart muscle for any given $\frac{\bar{\sigma}}{\bar{N}}$ diastolic volume varies directly with the nutrition index of its fibres and the $\overrightarrow{\bar{\sigma}}$ effective response of a poorly nourished myocardium to an increased load willo necessarily entail both acceleration of the rate of beat and a proportionately ${ }_{\vec{\omega}}^{\infty}$ greater degree of cardiac dilatation.

The above-mentioned physiological data indicate the heart's wide range of $\overrightarrow{\vec{\omega}}$ adaptability to variations in the volume of the venous return, equally also the $\frac{\text { o }}{8}$ beneficial effects of these variations on the healthy heart muscle and its outputo capacity.

Such considerations are amply confirmatory of the part played by the venous inflow in the maintenance of myocardial efficiency, and on them are based our views concerning the prescription of certain physical remedies, notably massageiv and physical exercises, in cardio-vascular derangements. These latter can, in the present context, be dealt with collectively, for it is generally acknowledged nowa-o days that massage and suitably planned exercises are potentially valuable at some stage or other in the course of all heart diseases, both functional and organic; in general terms the object of the treatment is restoration of circulatory efficiency $\tilde{c}_{\bar{D}}$ and of the outstanding factors participating in this process the following are $\vec{\theta}$ deserving of special mention:-

I. An increased venous return to the heart.

2. Improvement in the tone and contractile power of the heart muscle.

3. An increased cardiac output per beat, and

4. An accelerated blood supply to the coronary circuit.

The practical significance of these adjustments can be readily appreciated $;$ the heart, like other muscular structures, is capable of developing its optimumo efficiency when it is adequately trained, and physical treatment in the shape of massage and graduated exercises has come to be regarded as a rational and essential item in the management of heart disease and various of the circulatory derangements; its application is an especially material asset to patients recovering from acute carditis, to those suffering from persistent œdema, for which light local:massage is particularly valuable, and to those in whom obvious signs of returning compensation are observed following periods of failure in its various forms; undero such circumstances it is customary to prescribe a preliminary course of light generaß massage to the limbs and trunk and by degrees to introduce passive and easy? resistance movements at the larger joints; assuming satisfactory progress respira- $\rightarrow$ tory exercises may next be added to the daily programme, these in due course being followed by carefully graduated walking exercise at an easy pace, first on the level and later with the addition of gentle gradients; in a certain proportion of cases, more particularly those at or beyond middle age, the treatment outlinedu above may suffice to restore an effective circulatory reserve, but for the recupera- tion of children and young adults, further methods of physical therapy are avail able; suitably congenial amusements and outdoor games of various kinds can, as a rule, be chosen according to the patient's individual taste, his mentality, physica? development and the ascertained grade of his exercise tolerance, the one provisor being that patients must be warned to avoid all forms of strain which involve? holding the breath, their intelligent co-operation being at the same time ensured in? 
regard to the significance of breathlessness and of the clinical state conveniently referred to under the term " effort syndrome."

Further, it may be advisable in the less severe types of case to prescribe a graduated course of breathing and remedial exercises on the scheme originally formulated by Peter Henry Ling, who incidentally opened an institute in Stockholm in $18 \mathrm{I}_{3}$ for the training of teachers in gymnastics; a suitable programme of such exercises can be learnt without difficulty, and for those who prefer it numerous mechanical devices, as originally designed by Zander in I865 and since perfected by later workers, are nowadays available.

It remains that we should briefly consider the question of balneotherapy in heart disease, and more particularly the modern ideas relating to the remedial effects that are obtainable with the so-called carbonated baths which have gradually acquired universal repute. The essential basis of these baths is vaso-dilatation of the peripheral vessels, for the production of which certain explanations have been offered; the first of these is that the carbon dioxide gas exerts a local effect on the muscle fibres and nerve terminals in the walls of the vessels; secondly it has been suggested that the gas is definitely absorbed into the organism and that the benefit derived from the baths may to some extent depend upon direct stimulation of the myocardium and the intracardiac autonomic nervous system. The former of these theories, i.e., local stimulation by minute bubbles of gas on the surface of the body, is generally accepted, but the latter must still remain sub judice for there is as yet no convincing proof of the skin's permeability to thermal gases.*

Although, however, the problem needs further investigation we can in the meantime assess the clinical value and scope of carbonated baths on the physical developments they are known to achieve. Dilatation of the vessels, both superficial and deep, is an early mainfestation and a few minutes after the commencement of the bath the blood pressure, both systolic and diastolic, shows an appreciable decline; deceleration of the heart rate accompanies the fall in arterial tension, and it is authoritatively stated that the heart sounds become more clearly defined: otherwise the outstanding features are slow, deep breathing which is obvious during the course of the bath and the copious diuresis which follows it. A large number of cardiac and circulatory disabilities respond favourably to carbonated bath treatment, and it is especially indicated in cases of hypertensive heart disease, in endarteritis obliterans, in vaso-motor disturbances of the extremities, in certain of the arrhythmias, and in the relatively mild types of angina pectoris.

Apart from certain exceptional conditions, e.g., endocrine disorders, chronic renal disease, and eclampsia, the pathology of the large majority of hypertensive derangements is still a matter of speculation, but there is, nevertheless, a certain degree of similarity in cases of essential hypertension in that they all feature a generalised vaso-constriction; various factors, e.g., heredity, the circulation of pressor bodies, derangements of the vaso-constrictor and inhibitory nervous mechanisms have been held responsible for this development and the hypertensive effects of a generalised vaso-constriction are widely approved.

It is partly with a view to relieving vascular spasm that hydrotherapy, in the shape of carbonated baths, has been widely adopted in patients suffering from

* Investigations recently carried out by Ellman and Taylor discredit the theory that the skin is permeable to thermal gases in sufficient quantities to affect the gas tensions in the subcutaneous tissues (Ellman, P. and Taylor, H. J.: Jour. of Hygiene, xxxv, 3, Aug. 1935). 


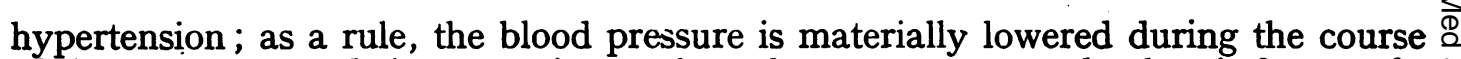
of the treatment and, in a certain number of cases, permanently, but it frequently $c$ happens that a reduction in tension is neither possible nor advisable, and the $\overrightarrow{\vec{c}}$ ultimate aim of the cure is the promotion of such a measure of equilibrium in $\overrightarrow{0}$ the circulation as will efficiently support the supernormal levels of pressure; in $\frac{0}{0}$ this respect a number of phenomena may be mentioned which testify to the cardiovascular adjustments induced by balneotherapy; the heart action is slowed, the $\mathbb{\AA}$ degree of its dilatation is reduced, its rhythm is regularised, the quality of its sounds is improved, the pulse pressure returns to the range of normality, the respiratory excursion becomes deeper and slower and a higher grade of renal efficiency is obtained; in conjunction with these signs there is obvious evidence of subjective improvement; palpitation and breathlessness are relieved to a varying extent, and functional disturbances, as headache, vertigo, tinnitus, paræsthesiæ, etc., lessen in intensity.

If, as frequently happens, the hypertensive state is associated with obesity. it may be advisable to supplement the carbonated baths with other forms of physiotherapy in the shape of massage, electrotherapy and graduated exercises, the customary restrictions in diet being at the same time observed; the programme of exercises is more especially designed with a view to improving posture and the range of respiration, and other forms of exercise, as walking, cycling, riding, etc., are gradually introduced, the patient's tolerance for increased activity being materially encouraged if he is provided with a well-fitting abdominal belt; a c convenient and suitable form of electrical treatment is faradism and, for the middle-aged hypertensive with a tendency to obesity, the Bergonie chair provides $\omega$ an ideal method of applying the current; under its influence rhythmic and painless contractions are induced in the muscles, their output of energy being regulated bo inserting appropriate resistances and by adjusting varying weights, sandbags, etc., so that the muscles are made to perform graduated exercises against suitably controlled resistances; in addition, moreover, to the local influences induced in the muscles, ergotherapy, so-called, is of value in that it stimulates the sluggish circulation to an improved level of efficiency, at the same time inducing an accelerated production of heat in the tissues and a proportionately increased basal metabolism, all of which are of material advantage in the successful management of patients with a tendency to obesity.

Another condition, endarteritis obliterans, is worthy of brief notice in the present context for it exemplifies to advantage the resources of purely physical $\frac{5}{3}$ treatment; the disability is characterised by an increased resistance in the vessels which depends partly upon structural changes and partly upon an excessive degree $\frac{\rho}{3}$ of vaso-constriction; the former of these is, admittedly, irremedial but the latter, in so far as it represents an exaggerated degree of sympatheticotonia, is capable of relief to a certain extent under suitable treatment; not the least important item of the latter is balneotherapy in the shape of carbonated baths, the specific actions of which include sympathetic sedation and relief from vascular spasm, the promo-o tion of vaso-dilatation and the development of an efficient collateral circulation, a $N$ slowing of the heart action and an improved diuresis; the value attaching to these $\underset{\omega}{\mathbb{N}}$ developments can be readily appreciated and, if such can be achieved, more $₹$ particularly in early cases, the prescription of carbonated bath treatment is amplyc justified; other physical remedies which have an established reputation in the therapeutic management of patients suffering from endarteritis obliterans are ? diathermy and radiant heat, both of which may contribute in the production of $\frac{0}{0}$ vaso-dilatation, collateral circulation, etc., sufficiently for the relief of such urgent $\mathbb{2}$ symptoms as pain, intermittent claudication and threatened gangrene. 
For certain other disease syndromes, notably angina pectoris, hypertension and Raynaud's disease, electrotherapy in the shape of diathermy has been prescribed during recent years and the results in carefully chosen cases have been encouraging. The value of the treatment depends essentially upon the heating effects of the deeply penetrating high frequency currents and the more important factors concerned include relief of vascular spasm, hyperæmia and an increased freedom of the blood and lymph circulation.

In cases of angina the so-called short wave diathermy is employed, the apparatus being so designed that it is capable of developing enormously high frequencies up to Ioo million cycles per second, the electro-magnetic waves so induced being relatively short as compared with those emitted by the less rapidly oscillating currents used in ordinary diathermy.

The earliest record of the use of short wave diathermy treatment in angina pectoris was communicated by J. Meyer in 1932 and a year later he published a series of twenty cases in collaboration with his colleagues Laubry and Walser, their more successful results being obtained in those patients whose anginal syndrome was the outcome of effort; Wallace Jones and John Hay investigated the value of diathermy in a group of forty patients at the Liverpool Royal Infirmary, and the opinion they formed was that the treatment improved the range of the exercise tolerance and increased the cardiac reserve, the most satisfactory results being observed in the high tension types of invalid; the electrocardiogram provides further confirmatory evidence of the good effect of diathermy in angina patients, various authors, notably Hyman (I933) having observed the development of an increased voltage of the $T$ waves following the treatment, such an alteration being interpreted as indicating improvement in the functional efficiency of the myocardium.

An increasing interest is attached to the possibilities of diathermic treatment in hypertension, and the subject has been ably epitomised by Wallace Jones in a recent work by Cumberbatch (I937); in addition to heating effects and local analgesia the author recognises two specific factors as instrumental in the reduction of supernormal blood pressure ; the first of these is a relaxation of vascular spasm, the second is a decreased viscosity of the blood and their combined effect is of obvious value in that tissue hyperæmia is encouraged, the peripheral resistance is diminished and the work of the heart is proportionately reduced.

It is fair to assume that diathermy treatment is theoretically justified in patients suffering with hypertension and, from the reports already available, its value in practice has been amply confirmed.

A series of cases analysed by Wallace Jones and John Hay (quoted by Cumberbatch) may be briefly mentioned in this connection; the series consisted of thirty-six patients, and of these the majority showed a definite and persistent lowering of pressure under diathermy treatment; certain of the patients were obviously unsuitable, symptoms of exhaustion, throbbing in the head, dizziness, etc., contraindicating further continuation of the treatment but more often than not the tolerance was satisfactory and it was significant that the majority of the successful cases improved subjectively both in regard to their physical capacity and their general feelings of well-being.

Cumberbatch, E. P. (1937) Diathermy, London.

Wallace Jones, H. and Hay, John.: Ibid. p. 253.

Same authors.: Ibid. p 251.

Hyman, A. S. (1933) Arch. Phys. Ther., xiv, No. 5.

Laubr y. Mayer and Walser (1933) Bull et Mémoirs de la Soc. des Hópit. de París, Vol. 49, p. 1290.

Meyer, J. (November 1932) Annales de l'institut. d'actinologie. 